\title{
MAGNETIC FIELD MEASUREMENTS OF PRINTED-CIRCUIT QUADRUPOLES AND DIPOLES*
}

\author{
W.W. Zhang", S. Bernal, P. Chin, R. Kishek, M. Reiser, M.Venturini, J.G. Wang, V. Yun \\ University of Maryland, College Park, MD 20742
}

\begin{abstract}
Printed-Circuit (PC) quadrupoles and dipoles have been designed and developed for focusing and bending a spacecharge dominated electron beam in the University of Maryland Electron Ring (UMER) [1], currently under development. Due to the rather small aspect ratio (length/diameter $<1$ ) of the magnets, the field quality, especially the nonlinear fringe field, has been a concern for the success of the UMER project. Extensive theoretical and experimental studies of the field structure of the magnets have been performed. Simple and precise methods for the magnetic field measurements of the PC magnets have been developed. In this paper, we present the various techniques and results of the measurements. The magnetic multipole components of quadrupoles and dipoles are determined from measurements with a long rotating coil. In addition, the integrated field of quadrupoles is obtained with the pulsed, taut-wire method. A comparison between the experimental results, theoretical analysis and calculation with a magnetics code, MAG-PC, is presented.
\end{abstract}

\section{INTRODUCTION}

A compact Electron Ring is being developed at the University of Maryland to study space charge effects and collective behavior of beams in a circular lattice [2]. The UMER has been designed to transport an electron beam of $10 \mathrm{keV}, 100 \mathrm{~mA}$, and $50 \mathrm{~ns}$ in a circular lattice of $11.5 \mathrm{~m}$ in circumference. The key components of the electron ring lattice are Printed Circuit (PC) quadrupoles and dipoles. The electron beam is focused by 72 PC quadrupoles and deflected by 36 PC dipoles. Table 1. lists the physical parameters of the PC quadrupoles and dipoles. These very short, air core magnets are made of current loops on flexible printed circuits [3]. This approach can meet not only the demand for beam transport in the electron ring, but also a significant reduction in cost. Nevertheless, the challenging problem is that the magnets have a rather small aspect ratio, resulting in highly nonlinear fringe fields. This concern has been addressed by extensive study of the field structure of the magnets in both theory and experiments.

Table 1: Physical parameters of PC magnets

\begin{tabular}{|l|c|l|}
\hline & Quadrupole & dipole \\
\hline Radius R & $2.79 \mathrm{~cm}$ & $2.872 \mathrm{~cm}$ \\
\hline Length L & $4.65 \mathrm{~cm}$ & $4.437 \mathrm{~cm}$ \\
\hline L/2R & 0.833 & 0.772 \\
\hline Grad (B Field) & $4.14 \mathrm{G} / \mathrm{cm}-\mathrm{A}$ & $5.22 \mathrm{G} / \mathrm{A}$ \\
\hline
\end{tabular}

\section{SPATIAL HARMONICS MEASUREMENT}

An accurate, widely used method of measurement of harmonics content in magnetic quadrupoles is the rotating coil [4]. A rectangular coil with dimensions 2.54 $\mathrm{cm}$ by $12.7 \mathrm{~cm}$ has been built [5]. It is used in such a way that one side of the coil is always positioned along the axis of the magnets, so the only relevant component of the magnetic field is $B_{\phi}$. The voltage waveform induced in the rotating coil, spinning at $6 \pm 0.001 \mathrm{~Hz}$, is digitized and fast-Fourier transformed in a scope. The oscilloscope is triggered by a synchronous signal related to the angular position of the rotating coil. Figure 1 is a photo of the basic setup.

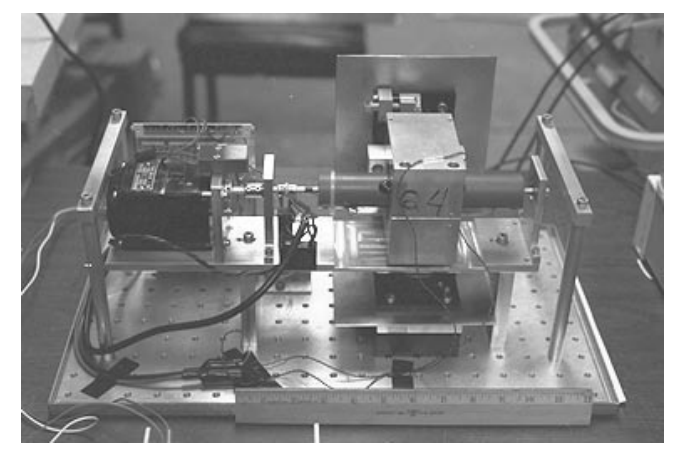

Figure 1: Rotating coil setup (without $\mu$-metal shield). The PC quadrupole is the block labeled "Q4" in the photo.

\footnotetext{
* Research supported by the U. S. Department of Energy

\# Email: wwz@glue.umd.edu
} 
The Earth's field and environmental noise are carefully compensated, since otherwise they produce a dipole term and other harmonic terms that affect the accuracy of measurement. To shield the effect of the Earth's field, a $\mu$ metal box is employed; the residual field is about $5 \mathrm{mG}$. The effect of random noise is reduced by averaging more than ten measurements. The first step is to adjust the PC magnet relative to the rotating coil to minimize the dipole component (for the quad), or the quadrupole component (for the dipole); a 3-axis support fitted with non-magnetic micrometer screws is used for this purpose, which makes quadrupole magnetic axis corresponding to the mechanical axis of the coil. In this way, the rotating coil allows us to determine the magnetic axis of each quadrupole relative to its mechanical axis to within $0.025 \mathrm{~mm}$. Figure 2 shows a typical spectrum from one quadrupole (with $\mu$-metal shield).

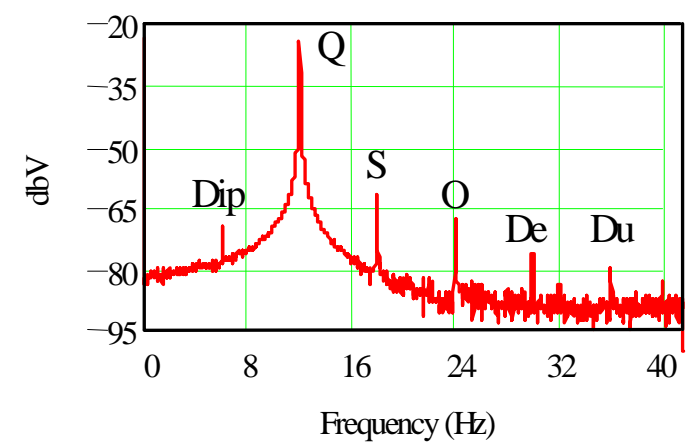

Figure 2: Harmonic spectrum of PC quadrupole:

Dip(ole), Q(uadrupole), S(extupole), O(ctupole), De(capole) and Du(odecapole).

Both theoretical analysis [6] and MAG-PC calculations show that the only high order harmonic term allowed in the UMER PC quadrupole is the duodecapole; for the PC dipole, the sextupole and decapole terms occur. The unexpected measured high order harmonic terms in both PC magnets can be traced to machining errors in the metal mandrels, small errors in assembly, and possible distortions of the printed circuit. The dipole term in the quad spectrum is due mostly to the residual Earth's field. Table 2 lists the measured harmonic multipole terms relative to the main component in both PC quadrupoles and dipoles. Each number is the average of thirty-two measurements.

Theoretical analysis [6] yields a duodecapole term for the PC quadrupole equal to $0.098 \%$, while MAG-PC calculations give $0.12 \%$, both in agreement with the measured value, within experimental error. The measured multipole terms have been used in single-particle studies and error analysis of the University of Maryland Electron
Ring [7]. They also provide important information for the mechanical alignment of the UMER.

Table 2: Measurement results

\begin{tabular}{|l|c|c|}
\hline Marmonic & Dipole(\%) & Quadrupole (\%) \\
\hline Dipole & 100 & 0.3 \\
\hline Quadrupole & 0.37 & 100 \\
\hline Sextupole & 0.51 & 0.3 \\
\hline Octupole & 0.14 & 0.2 \\
\hline Decapole & 0.11 & 0.19 \\
\hline Duodecapole & ----- & 0.15 \\
\hline
\end{tabular}

\section{MAGNETIC FIELD MEASUREMENT}

Because of the small size of the PC magnets, direct measurements with, for example, a Hall-probe gaussmeter is impractical. An alternative is to derive the field from integrated-field measurements using the pulsed taut-wire method [8,9]. Figure 3 shows the experiment setup.

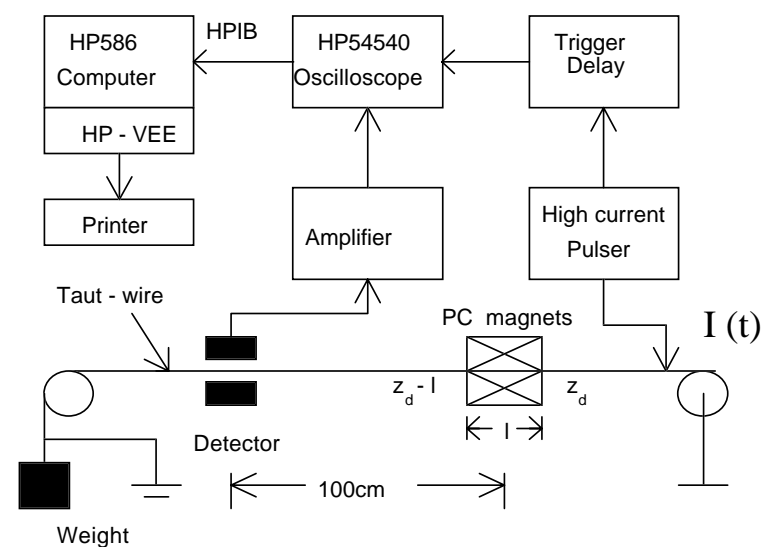

Figure 3: Setup for integrated magnetic field measurement with a pulsed taut-wire.

A current pulse (about $4 \mathrm{~A}, 300 \mu$ s at $0.2 \mathrm{~Hz}$ ) is applied to a long straight wire parallel to and some distance from the PC magnet axis. The Lorentz force on the wire produces a mechanical vibration that a photogate sensor turns into a voltage. The amplitude of the voltage waveform is proportional to the integral of the transverse magnetic field. In order to reduce the effects of room vibration and noise, the waveforms are averaged over at least 100 pulses. Since the Earth's magnetic field affects the motion of the wire, the waveform produced by the Earth's field alone is first obtained and then subtracted from the measurements. Figure 4 shows the result of a typical waveform after subtracting the Earth's field signal. 
To obtain an accurate value of the integrated magnetic field, a calibration is required. The simplest calibration method uses a set of small Helmholtz coils: the integrated magnetic field of the coils is carefully determined from measurements with both a gaussmeter and the taut-wire; thus the correspondence between amplitude of measured waveform and integrated field (i.e. number of volts per $\mathrm{G} \times \mathrm{cm}$ ) is established. Since the optical sensor has a limited linear area, the calibration is performed with care taken to avoid changing the sensor and taut-wire relative positioning used during the magnet measurements.

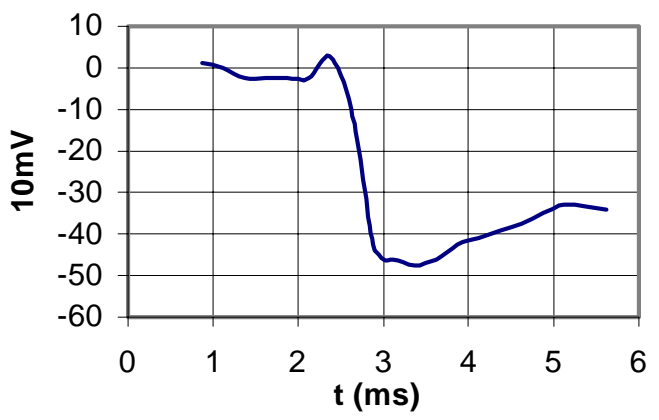

Figure 4: Typical signal from photogate sensor in the tautwire method.

After differentiation and normalization of a waveform similar to Figure 4, the normalized field profile of Figure 5 is obtained. The difference between experimental results and calculations is more pronounced in the tails, where the sensitivity of the taut-wire apparatus becomes a problem.

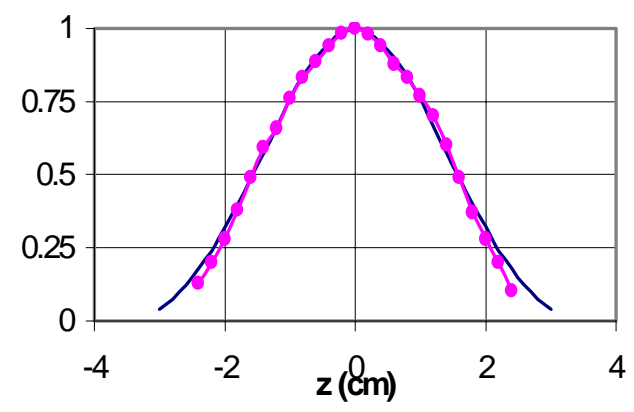

Figure 5: Normalized field profile of a PC quadrupole at $\mathrm{r}=0.45 \times$ quad radius and $\theta=45^{\circ}$. The solid line is the result of MAG-PC calculations.
An obvious improvement is to increase the peak current (without changing the pulse width) applied to the wire or the DC current applied to the magnet. This latter option, however, results in quadrupole heating.

The taut-wire method is also suitable for measuring the integrated magnetic field of pulsed magnets like the Panofsky quadrupole and the injector/extractor dipole intended for the Maryland Electron Ring [10].

\section{SUMMARY}

A rotating coil and a pulsed taut-wire apparatus have been used to measure the harmonic content and integrated magnetic field, respectively, of the PC magnets for the University of Maryland Electron Ring. These complementary measurements provide detailed information on the field quality of the small aspect ratio lenses, important for simulations of the beam dynamics as well as a realistic design of the mechanical lattice. The measurements are also significant on their own right, since the magnets are possibly the shortest (relative to their aperture), in existence.

The results of both the rotating coil and the pulsed-taut wire measurements are consistent with calculations with the iron-free magnetics code MAG-PC. The rotating coil results are also in fair agreement with theoretical predictions.

Studies similar to the ones reported here for DC magnets are planned for the pulsed elements of the UMER.

\section{REFERENCES}

[1] M. Reiser et al., these Proceedings

[2] M. Reiser et al, Fusion Engineering and Design, 32-33,293 (1996);

J. G. Wang et al., Particle Accelerator Conf., 1997, p. ????.

[3] T.F. Godlove, S. Bernal and M. Reiser, Particle Accelerator Conf., 1995, p. 2117.

[4] W.G. Davies, Nucl. Instr. and Meth. Phys. Res. A311, 399-436 (1992).

[5] Rawson-Lush Instruments Co., Inc. Acton, Massachusetts.

[6] M. Venturini, Ph.D. dissertation, University of Maryland, July, 1998.

[7] L.G. Vorobiev, X. Wu and R.C. York, Michigan State University unpublished Report, October, 1998.

[8] O. Shahal et al., Nucl. Instr. and Meth. Phys. Res. A259, 299-302 (1989).

[9] H.Nishihara and M.Terada, Journal of Appl. Phys. 41, 8, 33223324, (1970).

[10] Y. Li et al., these Proceedings. 